\title{
Outreach treatment was as effective as hospital treatment for comorbid intellectual disabilities and psychiatric disorders
}

van Minnen A, Hoogduin CA, Broekman TG. Hospital vs. outreach treatment of patients with mental retardation and psychiatric disorders: a controlled study. Acta Psychiatr Scand 1997 Jun;95:515-22.

\section{Question}

In patients with intellectual disabilities and psychiatric disorders, is outreach treatment as effective as hospital treatment?

\section{Design}

28 week randomised controlled trial.

\section{Setting}

Psychiatric hospital in Venray, the Netherlands.

\section{Patients}

50 patients (mean age 31 y, $76 \%$ men) who had a mild or borderline intellectual disability and were considered to require inpatient hospital treatment on the basis of the severity of their mental illness. Patients who required institutionalisation on legal grounds were excluded. Patients were recruited from referrals to a 48 bed Dutch psychiatric hospital.

\section{Intervention}

Patients were allocated to hospital treatment $(n=25)$ or outreach treatment $(n=25)$. Patients in the hospital treatment group received 24 hours/day of care consisting of psychopharmacological medicine, behavioural therapy, social skills training, education, and structured daily activities. Patients in the outreach treatment group received treatment in their home environment coordinated by a research team consisting of a psychiatrist, an educationalist, a social worker, 3 community psychiatric nurses, and a coordinator. A treatment strategy was determined by the outreach team and implemented in the patient's home environment. In addition to the patient focused interventions of the hospital treatment programme, motivational strategies were used to encourage caregivers to participate in supporting the patient and cooperating with the treatment.

\section{Main outcome measures}

Psychiatric symptoms as measured by the Psychopathology Inventory for Mentally Retarded Adults (PIMRA), the Reiss Screen for Maladaptive Behavior, and the Global Rating Scale for Improvement (GSI); family burden as measured by the 46 item Nijmegen Child-Rearing Situation Questionnaire; and hospital admissions. The costs of the interventions were also compared.

\section{Main results}

The PIMRA, Reiss Screen, and GSI assessments showed that psychopathology levels did not differ between the 2 groups at almost all trial points. Family burden did not increase from baseline among patients in the outreach treatment group. Psychiatric hospital admissions were prevented for $84 \%$ of patients in the outreach treatment group. The mean cost per patient per day was US\$268 in hospital treated patients and $\$ 148$ in outreach treated patients.

\section{Conclusion}

Outreach treatment of patients with intellectual disabilities in their home environment resulted in no increased psychiatric symptoms or family burden and minimal psychiatric hospital admissions.

\section{Source of funding: Ziekenfondsraad.}

For correspondence: Dr A van Minnen, Department of Clinical Psychology, University of Nijmegen, PO Box 9104, 6500 HE Nijmegen, the Netherlands. Fax +31 243615594

\section{Commentary}

This important study by van Minnen et al investigates a serious problem in service provision. People with intellectual disabilities and comorbid psychiatric disorders pose severe challenges to caregivers and to services generally as well as experiencing considerable distress themselves. It is essential to provide care for this group of people that is efficient, cost effective, and supports the maintenance of any gains achieved. As the authors point out, one of the problems of admission to hospital is that it may undermine the generalisation and maintenance of treatment effects because these will often involve socialenvironmental manipulations. Although the treatment of such problems in people's homes rather than in hospital is not a new concept, there have been no controlled studies identified with this population.

The study has several strengths, which include randomisation, the use of well established measures that were administered on a repeated basis, and a sound method of analysis. One problem is that randomisation was done before consent was sought and before the main exclusion criterion was established. This could have introduced some bias because the final study population included 50 of the 60 patients originally randomised to the group. A further problem is the dropout rate over the course of the study, with only 10 of the 25 patients in the hospital group and 17 of the 25 in the outreach group assessed at 28 weeks-the final assessment in the study.

The use of equivalence testing in the data analysis is particularly appropriate. ${ }^{1}$ This is a method which overcomes the problem of "trying to prove the null hypothesis" in showing that one interven- tion is as effective as another. The difficulty with the approach is in specifying what size of difference will be regarded as unimportant, but the authors make a reasonable choice in this regard.

Despite the dropout and the relatively short follow up period, this study is an important indication that outreach services can be as effective as admission to hospital, at lower cost and without increased burdens on carers. It shows that this approach merits further study and development.

Malcolm Adams, MA, MSc School of Health Policy and Practice University of East Anglia Norwich, Norfolk, UK

1 Rogers JL, Howard KI, Vessey JT. Using significance tests to evaluate equivalence between two experimental groups. Psychol Bull 1993; 113:553-65. 